\title{
PRÁCTICAS INCLUSIVAS EN EL NIVEL SECUNDARIO LA EXPERIENCIA DESDE LA PERSPECTIVA DE UNA ESTUDIANTE CON DISCAPACIDAD
}

\author{
Silvia Dubrovsky* \\ Universidad de Buenos Aires, Argentina \\ silviadubrovsky@gmail.com \\ Carla Lanza**. \\ Universidad de Buenos Aires, Argentina \\ carla.lanza1984@gmail.com
}

Recibido: 31/10/2018 Aceptado: 29/12/2018

\section{Resumen}

En el presente artículo se comparten algunos recortes de un proyecto de investigación cuyo propósito es indagar de qué modo se configuran las prácticas pedagógicas en el marco de lo que se ha denominado el paradigma de la inclusión, interpelando saberes, dispositivos y prácticas de la cultura institucional de las escuelas.

Tomamos como marco conceptual los desarrollos Vigotskyanos en tanto se propusieron, en su época, superar los reduccionismos de la psicología tradicional, al reconocer la unidad de los procesos intelectuales y afectivos, el origen social y cultural de los procesos psicológicos superiores, y el carácter mediado de la mente y la actividad humana (Vigotsky, 1930; Castorina, 2002; Del Cueto, 2015).

Este trabajo investiga, desde un enfoque etnográfico, el modo en que las prácticas denominadas inclusivas influyen en el desarrollo de los/as alumnos/as "integrados/as". Se toma para el análisis la perspectiva de una alumna "integrada" en relación a su experiencia escolar. Se propone el uso de la categoría perezhivanie como unidad de análisis para dar cuenta de la complejidad de las prácticas educativas en tanto el concepto relaciona los procesos y las leyes de desarrollo con el rol del ambiente social.

Palabras clave: Escuela secundaria - Inclusión - Discapacidad - Experiencia emocional Enseñanza.

\section{Abstract}

In this article we share some cuts of a research project whose purpose is to investigate how pedagogical practices are configured within the framework of what has been called the paradigm of inclusion, challenging knowledge, devices and practices of institutional culture of the schools.

We take as a conceptual framework the Vigotskyan developments as they proposed, in their time, to overcome the reductionisms of traditional psychology, by recognizing the unity of intellectual and

* Mg. en Psicología Educacional (UBA). Lic. en Ciencias de la Educación (UBA). Prof. Adjunta. Cátedra Teorías Psicológicas. Departamento de Ciencias de la Educación (UBA). Docente del Instituto Superior del Profesorado en Educación Especial y del ENS Nro 3 (CABA). Docente de la Especialización Superior Docente en Inclusión Educativa ENS Nro 6. UBA. Co-Directora del Equipo Educación y Psicopedagogía, CIDAC, SEUBE, FFYL-UBA.

** Maestranda en Psicología Educacional (UBA) Lic. y Prof. en Ciencias de la Educación (UBA).

Auxiliar docente de las cátedras Teorías Psicológicas y Análisis Sistemático de las Dificultades de Aprendizaje (UBA). Docente de Pedagogía y Educación Temprana en el ISFD N¹. Psicopedagoga en Hospital Vecinal de Gerli. Miembro del Equipo Educación y Psicopedagogía, CIDAC, SEUBE, FFYL-UBA. 
affective processes, the social and cultural origin of higher psychological processes, and the mediated character of the mind and human activity (Vygotsky, 1930; Castorina, 2002; Del Cueto, 2015).

This work investigates, from an ethnographic point of view, the way in which so-called inclusive practices influence the development of "integrated" students. The perspective of an "integrated" student in relation to her school experience is taken for the analysis. The use of the perezhivanie category is proposed as a unit of analysis to account for the complexity of educational practices as the concept relates the processes and laws of development to the role of the social environment.

Keywords: High school - Inclusion - Disability - Emotional experience - Teaching.

\section{Introducción}

\section{Los procesos de Inclusión en la escuela secundaria}

La inclusión en la escuela secundaria es parte de los objetivos y lineamientos prioritarios de la política educativa en el marco de la Ley 13688 de Educación de la Provincia de Buenos Aires, en consonancia con la Ley 26206 de Educación Nacional en la cual el Estado establece la obligatoriedad de dicho nivel.

Los lineamientos provinciales comprenden a la inclusión educativa en dos dimensiones constitutivas: la asistencia escolar y el acceso al conocimiento. La meta es entonces la inclusión que potencie el aprendizaje, la misma será plena en tanto garantice no solo el acceso y la permanencia de los jóvenes, sino efectivamente la apropiación de aprendizajes escolares socialmente significativos (Res. 4635, 2011).

Según datos estadísticos elaborados por la Dirección Nacional de Investigación y Estadística de la Calidad Educativa (DINIECE- Ministerio de Educación de la Nación), en los últimos años aumentó significativamente el número de alumnos con discapacidad que cursan su trayectoria escolar en las escuelas secundarias. Esto implica celebrar no solo las condiciones de democratización de acceso a la educación de sectores tradicionalmente excluidos, sino que convoca también a recrear, repensar, rediseñar la escuela como dispositivo de inclusión social.

En la Provincia de Buenos Aires, República Argentina, recientemente se ha sancionado la Res. 1664/17 en un intento de adecuar las normativas provinciales a la Resolución 311/16 del Consejo Federal de Educación de nuestro país. Dicha resolución distingue la integración escolar de la inclusión educativa y propone que esta última, en tanto derecho, requiere de prácticas institucionales y áulicas que la sostengan.

La posibilidad de tomar contacto con la cotidianeidad de las escuelas secundarias de la Provincia de Buenos Aires nos ha permitido observar una heterogeneidad de situaciones. La implementación de la normativa se traduce en prácticas que han adquirido distintas características en función de las concepciones de los actores sobre los procesos de enseñanza y aprendizaje, de los modelos e historias institucionales, y del devenir singular de cada trayectoria educativa. En ocasiones, las propuestas individuales diseñadas en cada proyecto de inclusión están pensadas desde las supuestas necesidades de cada joven, determinadas muchas veces por equipos y/o profesionales de diversa formación que generalmente provienen del área de salud. En este sentido resulta relevante analizar las prácticas de enseñanza que tienen lugar en las aulas.

En muchas oportunidades, hemos observado que los procesos "de inclusión" se desarrollan de acuerdo a lo que Parrilla Latas (1998, en Dubrovsky, 2001) ha denominado de "intervención sectorial". Es decir, sólo afectan al alumno "integrado" y no repercuten ni en sus compañeros ni en el sistema educativo, pues limitan la intervención y ponen la mirada casi exclusivamente en "los déficits del alumno". De este modo, pareciera interpretarse que la escuela inclusiva es la misma escuela de siempre, que incluye servicios de educación especial, produciéndose lo que Jacobo (2012) ha dado en llamar integración excluyente.

Este trabajo presenta avances de la investigación "Prácticas inclusivas. Análisis de la actividad de enseñanza en las aulas de escuela común con alumnos "integrados" reconocida por la FFYL-UBA por Res. 2830/16. La misma parte de desarrollos previos (Castorina y Dubrovsky, 2004) que argumentan que la teoría histórico-cultural ofrece herramientas teóricas y metodológicas para analizar las prácticas de enseñanza articulando las características de la práctica docente con los procesos de desarrollo de los alumnos (Castorina y Dubrovsky, 2004).

\section{Marco teórico La teoría histórico-cultural y las claves teórico- metodológicas para el análisis de las prácticas}

De la vasta producción teórica de Lev Vigotsky (1896-1934), de sus discípulos y de sus continuadores, resulta relevante plantear la fecundidad que el marco teórico histórico-cultural ofrece para la interpretación, investigación y reflexión de los procesos educativos y su potencialidad para el análisis de los procesos de inclusión escolar. Ya en otros trabajos hemos señalado la relevancia que Vigotsky le otorgaba a la enseñanza y su valor para el desarrollo cognitivo (Castorina, Dubrovsky, 2004) puesto que, como bien señalara el autor, "la enseñanza correctamente organizada conduce tras de sí al desarrollo infantil." (Vigotsky, 1999, p. 62)

Asimismo, Vigotsky plantea el desarrollo en términos de un proceso que no escinde lo psíquico de lo material ni lo individual de lo social (Vigotsky, 1932).

En los últimos años de su vida, Vigotsky realizó una revisión radical de importantes aspectos de sus 
primeros postulados a la luz de la filosofía monista de Spinoza. Si bien no tomó la teoría en su totalidad se propuso dar vida spinoziana a la psicología marxista. Tarea que, si bien no pudo terminar, tiene gran valor heurístico. La principal idea de Spinoza es que mente y cuerpo no son dos sustancias diferentes, de diferente tipo, sino que hay una sola sustancia que tiene diferentes atributos. Esto para Vigotsky constituye la antítesis del paralelismo, o sea del dualismo cartesiano. Lo biológico y lo cultural, lo individual y lo social, el pensamiento y el habla revelan las contradictorias e inseparables líneas de desarrollo (Jornet \& Roth, 2016). Basándose en Spinoza, plantea que desde el principio el problema de las pasiones -que en Descartes se establece como un problema fisiológico- es el de la relación existente entre pensamiento y afecto, el concepto y la pasión.

En varios de sus últimos escritos, Vigotsky hace referencia a la unidad de análisis que nos permite estudiar el desarrollo de los procesos superiores. Uno de los más relevantes es Una teoría de las Emociones (1933), que fue escrita prácticamente en simultáneo con Pensamiento y Lenguaje (1998). Aunque no llegó a desarrollarla por completo, es aquí donde Vigotsky propone una unidad de síntesis entre intelecto y emociones a la que llama perezhivanie. Se trata, en palabras de Zavershneva (2010) de una unidad integral de lo interno y lo externo, la personalidad y su contexto

En "La crisis de los 7 años" (1996) plantea:

El viraje fundamental que debe hacerse en el estudio del medio social es pasar de los índices

absolutos a los relativos, estudiar, ante todo, lo que significa para él. Cuál es su relación con las diversas facetas de dicho medio (Vigotsky, 1996, p.382).

En la traducción al español de ese texto en Obras Escogidas, se utiliza la expresión vivencia como traducción de perezhivanie, donde se la define como la unidad para el estudio de la personalidad y el medio.

En El problema de la edad, Vigotsky se refiere también al papel del ambiente del niño en su desarrollo. En el estudio del mismo, se ha considerado erróneamente al medio como algo externo en relación al niño, "como una circunstancia del desarrollo, como un conjunto de condiciones objetivas, independientes, sin relación con él, que por el simple hecho de su existencia influyen sobre el niño" (Vigotsky, 1996, p. 264). Más adelante, al autor señala que "la relación que se establece entre el niño y el entorno que lo rodea, sobre todo el social, es totalmente peculiar, específica, única e irrepetible para esa edad". A esta relación la denomina "situación social de desarrollo"(Vigotsky, p. 264)

La situación social de desarrollo representa el inicio de los cambios cualitativos en un determinado período del mismo y se refiere a la combinación de procesos internos y condiciones externas.

Señala Bozhovich (1989) que cada etapa en el desarrollo del niño en edad escolar se caracteriza por un conjunto especial de condiciones de vida y de la actividad de los alumnos y, por la estructura de las particularidades psicológicas que se forman bajo la influencia de estas condiciones. De allí el carácter dialéctico complejo entre las condiciones de vida del escolar y sus particularidades psicológicas. Las influencias del ambiente, señala Vigotsky, varían en dependencia de las propiedades psicológicas del niño formadas anteriormente. La unidad de análisis, para el estudio de la situación social de desarrollo, como señalamos, perezhivanie/ vivencia/ experiencia/ está representada, en un todo indivisible, por un lado por el ambiente, es decir lo experimentado por el niño, y, por otro, lo que el propio niño aporta a esta vivencia y que a su vez, se determina por el nivel ya alcanzado por él anteriormente. Señala también la autora que tras la vivencia se encuentra el mundo de las necesidades del niño, de sus aspiraciones, deseos, propósitos, en su complejo entrelazamiento y en su correlación con las posibilidades de satisfacción.

De este modo, para entender, analizar y profundizar en la comprensión de la relación entre la participación del estudiante en un proyecto de inclusión y su desarrollo, y, con la pretensión de alejarnos del marco epistémico de la escisión (Castorina, 2017) sostenemos la necesidad de analizar la situación social de desarrollo de los niños, niñas y adolescentes que cursan su escolaridad en el marco de estos proyectos de modo de superar los modelos que centran su análisis exclusivamente en la actividad del alumno/a y su maestro/a de apoyo.

Bozhovich (1989) realizó sus investigaciones con niños con dificultades de aprendizaje. Desde la perspectiva de análisis de las vivencias pudo determinar las condiciones y características que, en cada caso, generaron una serie de particularidades en su personalidad. En algunos casos marcadas por la posición del estudiante entre los que lo rodean y su interrelación con ellos y en otras por las dificultades para responder a las exigencias que plantea la actividad de estudio. De esta manera, se pudo analizar que las influencias del ambiente no siempre ejercen la misma influencia sobre el desarrollo. En la mayoría de los casos se vincula, entonces, con la posición del alumno entre los que lo rodean, especialmente de quienes depende su bienestar emocional. El gran valor que encontramos, entonces, en los aportes teóricos de la perspectiva histórico-cultural en el análisis de las prácticas inclusivas se vincula con la posibilidad de analizar estas prácticas, no solo desde la consideración de los ajustes necesarios, sino también, las vivencias, esto es, el modo en que estas experiencias en el aula, (entendiendo que es el aula de la escuela moderna, tradicional, la que intenta alojarlos/as) se refractan en el sujeto y configuran nuevas posibilidades de desarrollo o un obstáculo para el mismo.

La teoría nos permite ir más allá de los aspectos técnicos de la inclusión para adentrarnos en la experiencia emocional, es decir, en los efectos de las prácticas en el desarrollo subjetivo.

Considerar, la vivencia/la experiencia emocional (perezhivanie) que surge en el aula que denominamos inclusiva, colabora en la posibilidad de determinar qué tipo de influencia tendrá esta 
situación o este entorno en el alumno/a. Por lo tanto, no es ninguno de los factores en sí mismos (si se toman sin referencia al niño/a) lo que determina cómo influyen en el curso futuro de su desarrollo, sino que son los mismos factores los que permiten como si fueran un prisma refractar a través de ellos la experiencia emocional (perezhivanie).

Asimismo, el ambiente de los/as niño/as, jóvenes se modifica permanentemente e incluso cuando varíe poco, son los/as niño/as, jóvenes los que cambian en su proceso de desarrollo por lo que el rol y significado de los factores ambientales también han sufrido un cambio.

\section{Perezhivanie e inclusión educativa}

Las miradas reduccionistas y deterministas han puesto el foco en los tipos y características de la discapacidad para pensar los proyectos pedagógicos individuales o de inclusión (en adelante PPI). No obstante, las trayectorias de los adolescentes no tienen lugar en un vacío, estos proyectos se insertan en una situación que no es individual, sino social y colectiva. Desde nuestro marco teórico, entonces, sostenemos que pensar en la situación social de desarrollo es el único modo posible de correr la mirada exclusiva en el sujeto que aprende como único responsable, a partir de sus condiciones, de su trayectoria escolar.

Asimismo, podemos articular estos conceptos con otras propuestas teóricas de la teoría, en el entendimiento de que los sujetos en situación de discapacidad (1) participan, en la actualidad, en las aulas de las escuelas del nivel educativo correspondiente a su edad cronológica. Vigotsky sostuvo que era necesario identificar y diferenciar las condiciones secundarias de la discapacidad, de las primarias, en tanto que la persona con discapacidad puede estar actuando en un mundo social que tiene efectos formativos negativos sobre el desarrollo. En otras palabras, nos advierte sobre ciertos efectos provenientes de algunas propuestas pedagógicas que, centradas en el "defecto", generan o profundizan los problemas para la participación en el aula. Y, en consecuencia, tendrán impacto en su desarrollo cognitivo.

Vigotsky argumentó, en el caso de los niños con discapacidad, que es con la utilización de mediaciones, a través de los objetos culturales y herramientas que encuentran a disposición y pueden ser accesibles (por ejemplo el Braille o el lenguaje de señas) que los niños son capaces de auto-organizarse desde el "exterior" a través de "sistemas culturales simbólicos" (Knox y Stevens, 1993, p. 15 en Daniels, 2003). En esta línea, Karpov y Haywood (en Daniels, 2003) identificaron y describieron dos tipos de mediación: metacognitiva y cognitiva. La primera de ellas relacionada con la adquisición por parte del niño de instrumentos de autorregulación y auto planificación; y la segunda referida a la adquisición de instrumentos para la resolución de problemas en un dominio específico de conocimiento. De este modo, una buena enseñanza apunta a desarrollar la capacidad para relacionarse con los problemas de manera teórica y reflexionar sobre el propio pensamiento (Dubrovsky, 2006).

Es entonces que, partiendo de estos desarrollos teóricos, nos proponemos indagar: ¿Cuáles son las interacciones favorecedoras de la inclusión educativa? ¿Cómo influyen las características de la personalidad de cada adolescente? ¿Cómo se relacionan con las propuestas de enseñanza que ofrece la escuela secundaria? Es decir, ¿cómo se relaciona perezhivanie e inclusión?

\section{Metodología}

El proyecto de investigación está enmarcado en el paradigma de investigación cualitativa (Maxwell, 1996). Dentro de este paradigma se seleccionó el estudio de caso etnográfico (Neiman, en Vasilachis 2006). Se trabajó con una muestra intencional o teórica y se utilizaron fuentes primarias y secundarias. Desde una perspectiva interpretativa, Pérez Serrano (1994, p. 81) afirma que "su objetivo básico es comprender el significado de una experiencia" dado que permite el conocimiento de lo particular, de lo idiosincrásico, sin olvidar su contexto (Pérez Serrano, 1994). Por su parte los estudios de corte etnográfico posibilitan un trabajo reflexivo que permita describir y transformar la realidad social (Rockwell, 2009). El presente trabajo presenta los avances de su desarrollo en una escuela secundaria pública de gestión privada de la Provincia de Buenos Aires que trabaja en articulación con una escuela de la modalidad de educación especial pública de gestión estatal. Se presenta un estudio de caso y el análisis se centrará en los aspectos interpersonales, las propuestas de enseñanza y su relación con los procesos de desarrollo. De acuerdo a los objetivos propuestos se han realizado entrevistas, observaciones de clase y análisis de documentos.

Desde el punto de vista del análisis de las prácticas resulta central recuperar la voz de los actores. En este sentido, incluimos la voz de una estudiante porque consideramos que los/as estudiantes con discapacidad han sido históricamente o bien silenciados o bien hablados por otros. Es por ello que en este trabajo compartiremos algunos fragmentos de entrevistas y observaciones que resultaron significativas en tanto se focalizó especialmente en el estudio de las prácticas que incluye la mirada en los procesos interindividuales en el marco más amplio de los contextos interpersonales, sociales y culturales en los que se inscriben (Elichiry, 2010).

\section{Resultados}

\section{Presentación de la experiencia}

\section{La trayectoria de Martina en la escuela secundaria}

Desde que Martina comenzó 1er año de la escuela secundaria tuvo el acompañamiento en su trayectoria escolar de una maestra integradora (en adelante MI) (2) quien diseñó las configuraciones de 
apoyo necesarias en función tanto de la planificación de los docentes de cada asignatura como de las posibilidades de aprendizaje de la alumna. Sus informes describen a Martina como una adolescente sociable y locuaz que, a consecuencia de dos operaciones de tumores cerebrales, presenta desde la infancia dificultades en la elocución y comprensión del lenguaje, así como también en su memoria de trabajo. En el momento en que la conocimos, se encontraba cursando 6to año en una Escuela Secundaria de la Provincia de Buenos Aires en un grupo junto a 26 alumnos/as con quienes cursaba sus estudios desde el último año de la Escuela Primaria.

\section{-De las características personales y los procesos metacognitivos: "Yo quiero sacarme buenas notas"}

A partir de una de las entrevistas realizadas a Martina, podemos caracterizarla como una joven analítica y perseverante. Al respecto ella señala:

"Martina: Por ejemplo cuando estaba... eh... Ignacio (otro joven con proyecto de inclusión) que estaba en el otro año. Bueno con él no pudo estar más porque había cosas que ella (su maestra integradora) me contaba que a ella no le daban ni cinco de bolilla que la dejaba siempre haciendo las cosas sola, como diciendo que bueno es mi integradora entonces hace todo ella, no me importa nada, yo no hago... si... si .. lo hace ella. Pero yo no soy así, yo... a mí... bueno ella es lo que me quiso explicar de Ignacio pero... eh... yo siento que no soy así... yo soy muy terca (silencio).

E: ¿Qué quiere decir terca?

M: Eh... de sacarme buenas notas: quiero sacarme buenas notas. Si me saco malas notas me enojo, me pongo mal. Pero bueno aprendí que cualquiera se puede equivocar, que... cualquiera puede sacarse o un seis, o un cinco, como un cuatro, como un tres, como un dos, o como un uno" (Martina, J, entrevista personal, marzo 2017).

Martina tiene una mirada positiva de sí misma y nos cuenta, de algún modo, sobre su rol activo en la escuela. Sin duda esta actitud le ha permitido tomar otra posición en su proceso de aprendizaje. Su implicación y dedicación hacia las tareas escolares sumado a los apoyos que recibía han colocado a la joven en un rol realmente activo en el aula. Es por ello por lo que el análisis de los procesos inclusivos deja en evidencia que no son solo las condiciones individuales de Martina las que le permiten o no aprender si no que a lo largo de su trayectoria escolar se construyeron una serie de condiciones y situaciones con estos alumnos, estos docentes, su familia que crearon condiciones de posibilidad y así creemos lo vivenció Martina.

\footnotetext{
"E: ¿Cómo ves el trabajo que hizo P (Maestra Integradora) con los profesores? ¿Cómo trabajó ella todo este tiempo?

$\mathrm{M}$ : Eh, eh, $\mathrm{P}(\mathrm{Ml})$ bien pero los profesores... a ver.... por un lado $\mathrm{P}$ ya sabe lo que yo necesito, cómo hacer las pruebas, o cómo explicarme las cosas. Si no entiendo... explicármelo mejor. Pero yo no me cierra la palabra, o sea quiero decir que... no entiendo mucho para eso me tienen que volver a repetir lo que les dijeron a todos los chicos.

M: ella (la profesora de matemática) lo que está tratando de hacer es explicarle a los chicos y... y después explicarme a mí, que me siento y me explica y pero yo como que voy un poco más atrasada que los chicos.

$\mathrm{E}$ : Bueno pero la profesora, igual te da la clase, te explica ¿sentís que te pasa eso con todos los profesores?

M: Con todos no. Con todos no. Hay algunos que.... por ejemplo... ehmm el profesor de Lengua y Literatura, como que todo se lo explica a Paula (MI) pero yo me tengo que acercar para que él... para que él me explique... no me llama como la profesora de matemática que me dice vení Martina que te voy a explicar un tema nuevo. Que es lo que ella hace. No, yo me tengo que acercar a $P$ para preguntarle porque él... y además explica tan rápido que es como que se te hace más difícil todavía.

E: ¿Y con los otros profesores cómo es tu relación?

M: Por ejemplo la profesora de historia es sumamente... muy... además de hablar mucho, no sintetiza, no abrevia. No dice... y como que no le entendés si tenés que estudiar esto como... la cosa es que habla tanto, habla tanto, habla tanto y después no se le entiende tanto. Y... pero bueno son cosas normales viste.... (Martina, J, entrevista personal, marzo 2017).
}

Este diálogo con Martina pone en evidencia el conocimiento que ella misma tiene respecto de sus propios procesos cognitivos/ procesos de aprendizaje y de lo que ocurre en la clase.

Los procesos metacognitivos han sido señalados como el control automático de una ejecución, por ejemplo, la metacognición es para Ormrond (2008, p. 366) el conocimiento del individuo, de sus propios procesos cognitivos y de aprendizaje, así como la regulación de los mismos para mejorar el aprendizaje y la memoria. Así la autora sostiene que cuanto más sofisticado metacognitivamente es un estudiante, mejores son su aprendizaje y su rendimiento académico (Ormrond, 2008). Ahora bien, lejos de esta postura el planteo de Labarrere (2011) es que la metacognición sería el acceso consciente, el acceso deliberado al conocimiento de los procesos cognitivos y la regulación de la ejecución. "... Se trata, entonces, de establecer los nexos existentes entre conciencia en la ZDP (3) y desarrollo del funcionamiento cognoscitivo del estudiante; cuáles son las características del actuar consciente en la zona y del desarrollo aludido en tales circunstancias..." (Labarrere, 2011, p.142) 
En el fragmento presentado, Martina expone con claridad sus necesidades y tiene plena conciencia del modo en que funciona la dinámica escolar de la que participa. Puede reflexionar no solo respecto de sí misma y sus posibilidades sino, sobre todo, respecto de los otros. Puede hacer referencia tanto a los mediadores implementados por sus profesores como al modo en que los profesores dan respuesta a sus necesidades. De esta manera ella ubica que: su maestra integradora conoce cómo hacer las pruebas y cómo explicarle; que la profesora de matemática explica el tema una vez para toda la clase y luego se toma un tiempo para dialogar con Martina; que el profesor de Lengua explica rápido y no se dirige a ella, entonces Martina tiene que ir a su encuentro; que la profesora de historia "habla mucho, no sintetiza, no abrevia". Ella reconoce que necesita que le vuelvan a explicar. Primero realiza esta secuencia con su Ml y, ante su ausencia, toma "posición" y pareciera convertirse, ella misma, en su propia MI, buscando aquellas configuraciones de apoyo, en este caso, explicaciones más individualizadas y más pausadas para favorecer el registro de lo que se enuncia. Estos relatos dan cuenta de las posibilidades metacognitivas de la alumna, no siempre tenidas en cuenta por sus profesores y su MI. En algún sentido esta "conciencia" de los distintos estilos de enseñanza le permite solicitar y generar las mediaciones que necesita para aprender.

\section{- Cuando la configuración de apoyo es pensar con otros}

Históricamente se han utilizado diferentes conceptos para pensar las prácticas inclusivas. Se las ha llamado, entre otras, "necesidades educativas especiales" o "adaptaciones curriculares" pero en cualquier caso, el énfasis de dichas prácticas está puesto en las condiciones individuales de los sujetos. Ahora bien, a partir de la sanción de la Convención Internacional por los Derechos de las Personas con Discapacidad se comenzó a utilizar el término "configuraciones de apoyo", concepto que desde nuestra perspectiva da cuenta del estado indivisible entre las condiciones individuales y sociales permitiendo pensar aquellas redes, relaciones, mediaciones que el medio debe ofrecer para que los estudiantes puedan aprender. Con este marco es que analizaremos dos fragmentos de una observación de clase de la asignatura Proyecto de Investigación en Ciencias Sociales. En el primero, se observa la interacción entre Martina y su docente:

Martina le dice a la Profesora que su maestra integradora le comunicó que ella no tenía que hacer la misma tarea que el resto porque era muy difícil si la docente podía darle algo para leer y subrayar. La docente le responde que ella ya habló con su Ml y le explicó que la entrevista es una tarea que constaba de muchas partes y que como era un trabajo en equipo podían dividirse. Martina insiste en que no le va a salir. La docente se acerca al grupo de trabajo de Martina, conversa con ella y sus tres compañeras. Se retira y comienzan a trabajar todas en la misma actividad. (Registro de campo, noviembre 2016).

En el segundo, la profesora a cargo de la materia, interactúa con la observadora en los siguientes términos:

... hablé varias veces con la Ml y la Directora porque la Ml decía que Martina no podía hacer esa tarea. Le expliqué en qué consistía y que yo iba a insistir porque aprender a hacer una entrevista es más que un contenido de mi materia, tienen que ver con poder conversar con otros y desde mi punto de vista es algo de lo que Martina podía sacar provecho... (Docente, entrevista personal, noviembre 2016).

Estos recortes nos permiten sostener que Martina tiene internalizado un lugar de alumna "distinta" dentro de la clase. Sin embargo, la docente intenta construir un lugar alternativo, un espacio de confianza, confía en Martina y en sus posibilidades de participación en la actividad de la clase.

Esta docente que invita al trabajo cooperativo, intenta mover a la alumna del lugar del "no saber" y apuesta a su desarrollo, a sus posibilidades. En vez de ubicarla en el lugar de una alumna portadora de un déficit, desde su espacio curricular, procura habilitar el aprendizaje, o por lo menos abre la posibilidad a que ello suceda. Labarrere (1996) señala:

Es muy importante la posición de los sujetos como modelos durante su interacción; incluso reconoce que una de las formas de producir desarrollo en la zona es, introducir personas cuyos patrones de comportamiento son percibidos, reconocidos o, mejor, resignificados por el aprendiz... (Labarrere, 1996, p.6).

Es decir, que más allá de la ayuda instrumental es necesario tener en cuenta el componente afectivo. Esta afirmación nos resulta sumamente interesante en dos perspectivas. Por un lado, como sostiene Labarrere, en la interacción en la ZDP, la reestructuración cognitiva debe ser solidaria de la reestructuración emocional. Por otro, nos permite reafirmar la relación que debe establecerse entre las configuraciones de apoyo que se diseñan en los proyectos de inclusión y el establecimiento de vínculos sostenidos en los aspectos afectivos emocionales de los sujetos.

Desde la perspectiva de la teoría histórico-cultural, queda suficientemente explicitado que el aprendizaje es entendido como una actividad social y además plantea al lenguaje como el instrumento mediador por excelencia. Por lo tanto, al asumir el origen social de los procesos psicológicos superiores, abandonamos la idea de que los mismos son producto del desarrollo individual del sujeto sin desconocer su lugar protagónico. Por eso, el diseño de la actividad de enseñanza del docente se vuelve nodal para comprender los procesos de aprendizaje y en pos de ellos diseñar estrategias 
inclusivas que garanticen el derecho a la educación de todos/as. Este proceso no será posible en el marco de la teoría si no se produce, un entrecruzamiento de las subjetividades de quienes participan, sus motivaciones y necesidades (Labarrere, 1996).

\section{Conclusiones}

La introducción de la obligatoriedad de la escuela secundaria en Argentina significó el ingreso a este nivel educativo de muchos/as jóvenes que históricamente habían quedado por fuera y la responsabilidad del sistema educativo de generar condiciones de permanencia y de apropiación de conocimientos socialmente significativos. Asimismo, en el caso de los/as jóvenes con discapacidad, la sanción de la Convención Internacional por los Derechos de las Personas con Discapacidad y las reglamentaciones jurisdiccionales en relación a su inclusión en las escuelas llamadas "comunes" así como los cambios en las condiciones de acreditación y promoción, permitió su acceso a las escuelas secundarias. A lo largo de este artículo hemos intentado dar cuenta del modo en que se despliega cotidianamente la trayectoria educativa de los jóvenes con discapacidad que cursan sus estudios secundarios en una escuela común.

Asimismo, nos hemos propuesto este análisis a la luz de los aportes teóricos de la teoría histórica cultural de Lev Vigotsky, especialmente aquellos desarrollos conceptuales correspondientes al último período de su vida. El giro spinoziano de esta etapa se evidencia en la reformulación de la unidad de análisis del estudio del desarrollo del psiquismo que dé cuenta de la unión entre los elementos cognitivos y afectivos, entre lo social y psicológico y, que dicha unidad adquiere características peculiares en cada momento del desarrollo en función de las particularidades de las relaciones dialécticas entre los elementos que conforman las dualidades mencionadas conformando la situación social de desarrollo. Dicha unidad de análisis es perezhivanie.

Nos propusimos analizar, a partir del registro de observaciones de un estudio de caso (Martina) en diferentes horas de clase en su escolaridad secundaria, así como de las conversaciones con ella y con sus docentes, cómo se construye la experiencia (perezhivanie) cuando nos referimos a los procesos de desarrollo de niños/as y jóvenes que transitan su escolaridad en el ámbito de la escuela común.

En primer lugar, resaltamos la potencia de los procesos metacognitivos en relación a la posibilidad de participar y demandar a los docentes propuestas de enseñanza que en este caso no dejen a Martina por fuera de lo que ocurre en el aula. A partir de los fragmentos analizados fue posible conocer los recursos y estrategias que a modo de "mediadores" operaron, en el caso de Martina, en la ZDP. Si tenemos en cuenta, muy especialmente, el reconocimiento que tiene Martina de los diferentes modos de interacción que se suscitan en el aula con los diferentes profesores, es decir, el conocimiento de las acciones de enseñanza necesarias para mejorar su comprensión y su aprendizaje, es posible pensar, entonces que una tarea relevante a desarrollar en el aula sería generar condiciones para acompañar al docente del aula que le permitan identificar cuando la Ml- en tanto mediadora- puede o debe retirarse de la escena y el valor que este movimiento tendrá en el aprendizaje de Martina.

En segundo lugar, hemos analizado el modo en que la enseñanza puede traccionar el desarrollo cuando se hace lugar a las posibilidades de aprendizaje y el docente no se paraliza ante los sentidos que se construyen alrededor de una condición que podría atribuírsele a una niña con determinada patología o en situación de discapacidad, Cuando el/la docente se ubica en el espacio de posibilidades se ponen en marcha una serie de movimientos y acciones que promueven y hacen lugar al derecho a la educación.

En tanto existe una distancia entre el modo en que la profesora de la materia observada apuesta por las posibilidades de Martina y el modo de actuar de la Ml, habría "dos modos de ser Martina": la que puede, según la Profesora, interactuar y resolver las actividades junto a sus pares y aquella que "no lo puede hacer". Numerosas miradas respecto al análisis de la ZDP resaltan el hecho de que se supone un sujeto pasivo respecto de la ayuda que recibe del otro. Resulta sumamente interesante el análisis de Labarrere (1996) para interpretar el lugar en el que muchas veces son colocados los alumnos con apoyo a la inclusión. En tanto la ayuda es la forma fundamental de interacción en la zona, sostiene el autor que rara vez se le confiere al alumno la posibilidad de determinar si debe ser ayudado o no. La MI pareciera ubicarse dentro de esta perspectiva, en tanto decide, coloca de su lado, exclusivamente, las decisiones sobre qué ayudas necesita Martina. Para Labarrere (1996) la introducción forzosa de ayuda solo genera pasividad y dependencia exagerada de lo externo. De este modo, es importante tener en cuenta que al actuar en la zona se puede operar de modo de producir desarrollo -tal como ocurre en las interacciones de Martina con su profesora- o, por el contrario, se instale una suerte de inmovilidad que lo frene. Martina oscila entre estas dos posiciones en las que es colocada y desde las cuales es mirada.

Si bien no tenemos referencias sobre qué ocurrió en los años de escolarización previa al inicio de nuestro trabajo de investigación, sí podemos reafirmar, a partir de esta experiencia, que las configuraciones de apoyo no son solo tarea privativa de los maestros integradores. El rol desempeñado por el/la profesora, la interacción con sus compañeros/as, entre otras dimensiones, configuran el modo en que esta situación se refractará en ella y construirá y desplegará posibilidades de aprendizaje y desarrollo.

En este sentido sostenemos que perezhivanie es la unidad de análisis que permite pensar ya no en términos de causas y efectos sino a Martina y el ambiente como dos manifestaciones de la misma "sustancia". Desde la perspectiva de Spinoza, no tiene sentido pensar en perezhivanie en forma independiente de la situación social (Jornet and Roth, 2016), es decir, separar la singularidad de Martina que constituye la situación social de desarrollo, de las interacciones que mantiene con sus docentes y compañeros. Esto da cuenta de la complejidad que requiere el análisis de los proyectos de inclusión en 
tanto implica considerar sus múltiples dimensiones.

En las páginas precedentes, hemos intentado ofrecer algunas líneas de pensamiento para superar el modelo tradicional de adaptaciones curriculares, en tanto "recortes de contenido" y se ha brindado una perspectiva teórica que se ubica en la zona de posibilidades y de construcción de contextos donde sujetos, conocimientos, vínculos afectivos, confianza, diálogo, enseñanza y desarrollo son sólo algunos de los elementos que configuran una práctica que pueda considerarse realmente inclusiva.

La categoría perezhivanie, propuesta por Vigotsky como unidad de análisis, aunque superficialmente explicitada permite integrar la experiencia en el desarrollo subjetivo que implica, a su vez, una afirmación externa o aceptación de los otros, por ejemplo, de los docentes.

En la situación analizada en el marco de la investigación, el desarrollo se vuelve evidente/tangible en la cotidianeidad del aula cuando podemos comprender el sentido de los aprendizajes, las estrategias y herramientas simbólicas y materiales que los/as adolescentes elaboran y negocian con otros para construirlos.

Por último sostenemos que esto fue posible porque los actores involucrados comprendieron que no se es alumno o docente si no se está atravesado por la experiencia escolar y que la misma está constituida por condiciones institucionales, procesos cognitivos y afectivos absolutamente singulares en una determinada situación social de desarrollo.

\section{Notas bibliográficas}

(1) Vigotsky no hablaba explícitamente de discapacidad, concepto más moderno, sino de una disciplina que denominó defectologia, Vigotsky, 1996.

(2) Las maestras integradoras pueden ser tanto profesionales del área de salud como del área de educación. Su función es acompañar las trayectorias escolares de niños y jóvenes con discapacidad en las escuelas de nivel inicial, primario, secundario. No pertenecen al sistema educativo si no que provienen del área de salud, para contar con esta prestación de apoyo a través de Obras Sociales y de Medicina prepaga es requisito que los niños y jóvenes cuenten con un certificado de discapacidad. En la Ciudad Autónoma de Buenos Aires esta figura recibe el nombre de Acompañante Personal No Docente.

(3) Vigotsky introduce el concepto de zona de desarrollo próximo entendido como “... la distancia entre el nivel real de desarrollo determinada por la capacidad de resolver independientemente un problema, y el nivel de desarrollo potencial, determinado a través de la resolución de un problema bajo la guía de un adulto o en colaboración con otro compañero más capaz..." (Vigotsky, 1978, s/p).

\section{Referencias bibliográficas}

- $\quad$ Ainscow, M. (1999). Understanding the development of inclusive schools, Falmer, Londres.

- Aizencang, N y Bendersky, B. (2009). La Inclusión, ¿una problemática actual? en Escuelas y prácticas inclusivas. Intervenciones psicoeducativas que posibilitan. Manantial, Buenos Aires.

- Booth, T. \& Ainscow. M. (2002). Index for inclusion (2nd ED). Developingleaning and participation in schools $\left(2^{\mathrm{a} e d}\right)$. Manchester: CSIE [trad. Guía para la evaluación y mejora de la educación inclusiva. Consorcio Universitario para la Educación Inclusiva. Madrid: consorcio.educacion.inclusiva@uam.es.

- Bozhovich, L. (2009). "The Social Situation of Child Development". Journal of Russian and East European Psychology, 47(4), 59-86.

- Castorina, J. A. (2002). "El Impacto de la Filosofía de la Escisión en la Psicología del Desarrollo Cognoscitivo". Psique, 11 (1), 15-27.

- Castorina, J.A y Dubrovsky, S. (2004) "La enseñanza y la teoría psicológica socio- histórica. Algunos problemas conceptuales". En: José Antonio Castorina y Silvia Dubrovsky (comp.) Psicología, Cultura y Educación. Perspectivas desde la obra de Vigotsky. Noveduc, Buenos Aires.

- Castorina, A. (2017). "Los obstáculos epistemológicos en la constitución de la psicopedagogía 25 años después". En Filidoro, N; Dubrovsky, S; Rusler, V; Lanza, C; Mantegazza, S; Pereyra, B; Serra, C. (Comp.) Pensar las prácticas educativas y psicopedagógicas. Editorial Facultad de Filosofía y Letras, Buenos Aires.

- $\quad$ Daniels, H. (2003). Vygotsky y la pedagogía. Paidós, Barcelona.

- Del Cueto, J. (2015). "Dos nociones para un enfoque no escisionista de las emociones y la afectividad: situación social del desarrollo y vivencia en Vigotsky”. Perspectivas en Psicología, Vol.12, №1. Mayo 2015, 29-35.

- Dubrovsky, S. Navarro, A; Rosenbaum, Z. (2001). llusiones y verdades acerca de la integración en la escuela común. Dirección de Investigaciones. Secretaría de Educación Buenos Aires. Dubrovsky, S. (2005) La integración escolar como problemática profesional. Novedades Educativas, Buenos Aires.

- Dubrovsky, S. (2006). "La búsqueda de respuestas posibles de la teoría socio-histórica. de Vigotsky a las nuevas preguntas de la educación especial actual". Espacios en Blanco. Universidad Nacional del Centro de la Provincia de Buenos Aires. Nro. 16, 187-210.

- Elichiry, N. (2010). Aprendizaje y contexto: contribuciones para un debate. Manantial, Buenos Aires.

- Jacobo, Z. (2012). Las paradojas de la integración/exclusión en las prácticas educativas. Noveduc, Buenos Aires.

- Jornet, A \& Roth, W (2016). Perezhivanie-A Monist Concept for a Monist Theory, Mind, Culture, and Activity, DOI: 10.1080/10749039.2016.1199703

- $\quad$ Karpov, Y V y Haywood, H. (1998). American Psychologist, Vol 53(1), Jan 1998, 27-36.

- Knox, J. \& Stevens, C. (1993). "Vygotsky and Soviet Russian Defectology: An Introduction. In: The Collected Works of L. S. Vygotsky". Volume 2: The Fundamentals of Defectology (Abnormal Psychology and Learning Disabilities). Editors of the English translation: R. W. Rieber and A.S. Carton. Plenum Press, New York. 
- $\quad$ Labarrere Sarduy, A. (1996). "Interacción en ZDP: qué puede ocurrir para bien y qué para mal”. Ponencia presentada a Pedagogía '97, La Habana.

- Labarrere, A. (2011)."Funcionamiento cognitivo y desarrollo en ZDP. Pensamiento Educativo". Revista de Investigación Educacional Latinoamericana, 32(1), 141-15. Disponible en: http://pensamientoeducativo.uc.cl/index.php/pel/article/view/250/528

- $\quad$ Labarrere Sarduy, A (2016). "Zona de Desarrollo Próximo como eje del desarrollo de los estudiantes: de la ayuda a la colaboración". Summa Psicológica UST 2016, Vol. 13, № 1, 45-56. Vol 13. Número 1-293.

- Leontiev, A. (1983). Actividad, conciencia, personalidad. Pueblo y educación, la Habana.

- Leontiev, A. (1995). El desarrollo del psiquismo. Capítulo: El hombre y la cultura. Ed. Akal Editor, España.

- Maxwell, J. (1996). Qualitative Research Design. An interactive approach. Sage, Londres.

- Neiman, G; Quaranta, G. (2006). "Los estudios de caso en la investigación sociológica", en Vasilachis de Gialdino (comp.) Estrategias de investigación cualitativa. Gedisa, Buenos Aires.

- $\quad$ Ormrod, J, (2005). Aprendizaje humano. 4ta. Ed. Madrid, España: Pearon Printice Hall.

- Parrilla Latas, A. (1998). Apoyo interno, modelos y funciones En: Apoyo a la escuela un proceso de integración. Mensajero, Bilbao.

- Pérez Serrano, G. (1994). Investigación cualitativa. Retos e interrogantes. I.Métodos. Editorial La Muralla, S.A. Madrid.

- Rockwell, E. (2009). La experiencia etnográfica: historia y cultura en los procesos educativos. Paidós, Buenos Aires.

- Vigotsky, L. (1930-1997). Sobre los sistemas psicologicos. En Obras Escogidas (Tomo I, pp.71-93). Visor, Madrid.

- Vigotsky, L. (1932). El problema de la edad. En Obras escogidas (Tomo IV, pp. 251-273). Visor, Madrid.

- Vigotsky, L. (1933). Teoría de las emociones. Editoril Akal, Madrid.

- Vigotsky, L. S. (1933/2006). La crisis de los siete años. En Obras escogidas (Tomo IV, pp. 377-386).Visor, Madrid.

- Vygotsky, L. (1994)."The problem of the environment". En R. Van der Veer\&. J. Valsiner (Eds.), The Vygotsky reader (338-354). Blackwell, Oxford. (Trabajo original publicado en 1935).

- Vigotsky (1996). Obras escogidas, Vol. 4. Visor, Madrid.

- Vigotsky L. (1998). Pensamiento y Lenguaje. Editorial Pueblo y Educación, La Habana, Cuba

- Zavershneva, Elu. (2010). "The Vygotsky Family Archive: New Findings Notebooks, Notes, and Scientific Journals of L.S. Vygotsky" (1912-1934) Journal of Russian and East European Psychology, vol. 48, no.1, January-February 2010, 34-60.

\section{Normativas}

Ley $\mathrm{N}^{\circ}$ 13688. Ley de Educación de la Provincia de Buenos Aires, Decreto Promulgación: 1296/2007. Fecha B.O.: 10/07/2007.

Ley N 26206 de Educación Nacional de la República Argentina 14 de diciembre de 2006.

Res. 4635. La inclusión de alumnos y alumnas con discapacidad con proyectos de integración en la Provincia de Buenos Aires. Dirección General de Cultura y Educación. Provincia de Buenos Aires. 2013.

Resolución N¹664/2017. Dirección General de Escuelas, Provincia de Buenos Aires.

Resolución №311/2016, Consejo Federal de Educación. 